Revue d'histoire de l'Amérique française

DEVUE D.HISTOIRE DE L'AMÉRIQUE FRANÇAISE

\title{
Louisiane et langue française
}

Jeanne Grégoire

Volume 15, numéro 1, juin 1961

URI : https://id.erudit.org/iderudit/302095ar

DOI : https://doi.org/10.7202/302095ar

Aller au sommaire du numéro

Éditeur(s)

Institut d'histoire de l'Amérique française

ISSN

0035-2357 (imprimé)

1492-1383 (numérique)

Découvrir la revue

Citer cet article

Grégoire, J. (1961). Louisiane et langue française. Revue d'histoire de l'Amérique française, 15(1), 59-63. https://doi.org/10.7202/302095ar d'utilisation que vous pouvez consulter en ligne.

https://apropos.erudit.org/fr/usagers/politique-dutilisation/ 


\section{LOUISIANE ET LANGUE FRANÇAISE}

Le voyage de liaison française qu'ils firent en Louisiane, en janvier 1955, a permis à un groupe de Canadiens et d'Acadiens de se rendre compte que les Louisianais sont restés Français de cœur et d'esprit par leur foi et leurs coutumes, même s'ils parlent peu la langue de leurs ancêtres; ils sont aussi patriotes que les gens d'autres nationalités auxquels ils sont mêlés. «La tradition et le souvenir de leurs ancêtres français ne gênent pas leur loyalisme américain; ils respectent et aiment leur héritage français; ils le conservent dans leur vie quotidienne », affirment certains curés, qui continuent de prêcher la doctrine catholique en français et de dispenser l'enseignement du catéchisme en français dans plusieurs paroisses.

Ce qui étonne surtout le visiteur, c'est de trouver très peu de français dans la ville reine de cet état, la Nouvelle-Orléans, qui, plus que tout autre centre de la Louisiane, eut des origines purement françaises. Fondée par Bienville, en 1718, la NouvelleOrléans a été inondée de littérature française par le moyen de revues et de journaux, quotidiens et hebdomadaires. "Que de journaux! ils éclosent comme des champignons et meurent comme des mouches », écrivait Edward Larocque Tinker, auteur d'une bibliographie des journaux et périodiques de langue française en Louisiane. Bibliographie où j'ai puisé plusieurs titres, dont voici la nomenclature: le Moniteur de la Louisiane, fondé par Duclot en 1794; la Gazette de la Louisiane, dont la rédaction dans sa partie française était signée du pseudonyme «l'Hermite du Bayou » (1817) ; la Lyre louisianaise (1817), de GuillaumeAuguste Montmain; l'Abeille, qui prit naissance en 1827 et qui, de quotidien, devint hebdomadaire en 1917, pour disparaître en 1923, soit après environ un siècle de vie, battant ainsi le record d'existence des publications du même genre en Louisiane; l'Album littéraire, périodique fondé en 1843 et rédigé par un groupe 
d'hommes de couleur sous la direction de Jean-Louis Marciacq; le Franc-Parleur, d'Auguste Crébassol; l'Entracte; la Louisiane; la Chronique; l'Orléanais; le Meschacebé; les Cenelles, journal fondé en 1845, sous la direction d'Armand Lanusse; le Propagateur catholique; le Vigilant; l'Encan (journal des ventes); le Pionnier de l'Assomption.

Le premier journal publié à Saint-Martinville, berceau du peuple acadien en Louisiane après la migration du Maryland, fut la Gazette des Attakapas, fondée en 1824. Vint ensuite le Créole, édité par Alexandre Barde. Le Courrier du Tèche, publication mi-anglaise et mi-française, fut imprimé durant la guerre civile au revers du papier-tenture, en raison de la rareté du papier-journal. On en trouve encore un exemplaire exposé à la vue des visiteurs, au musée acadien de M. André Olivier, conservateur de la plus grande partie des documents historiques de Saint-Martinville et par conséquent des premiers Acadiens de cette contrée, considérée comme le véritable cœur du pays d'Évangéline.

Toutes ces publications ont été un précieux apport pour la langue française en Louisiane. Des événements se succédèrent qui vinrent altérer ou plutôt entraver cet essor merveilleux. De 1829, date de l'arrivée des Américains à la Nouvelle-Orléans, jusqu'à 1841, la situation changea beaucoup. Graduellement, la langue française disparut de la Législature, des tribunaux et du commerce. En 1858, un député du nom de Sterling proposa de supprimer la publication en français des documents officiels, proposition demeurée sans lendemain; mais en 1862, le général Butler, chef des nordistes qui vinrent occuper la Nouvelle-Orléans, porta un coup fatal à la langue française en l'attaquant à la racine même, par la suppression totale des écoles publiques françaises. Les créoles réagissent et font une tentative de survie en ouvrant des écoles privées, mais cet effort ne fera que ralentir, jusque vers la fin du siècle, le déclin progressif de la langue française.

Après ces soubresauts causés par la législation et la guerre civile, on ne voit apparaître que quelques journaux français: 
la Renaissance louisianaise, fondée vers 1861 par Hiriart et qui n'a vécu que dix ans; le Carillon, de Forester Durel; l'Union; la Tribune et quelques autres journaux sous la direction d'hommes de couleur; le Progrès; le Louisianais, fondé et rédigé uniquement par Jean Sylvain Gentel, et qui vécut de 1865 à 1881; la Démocratie française; l'Avenir, l'Équité, fondée par Charles Testut après 1865; l'Observateur louisianais, fondé par l'abbé Rougé en 1892 et qui cessa de paraître en 1897; le Pionnier. On constate qu'en 1896, il ne se trouve plus que six journaux de langue française. La marche vers la déchéance est rapide.

En 1879, une sensible amélioration dans la législation, qui permet l'enseignement du français dans les écoles primaires des paroisses de langue française, fait naître une lueur d'espoir. Un médecin français du nom d'Alfred Mercier fonde une sorte d'académie, nommée l'Athénée, dont les membres s'emploieront à diverses publications en plus de rédiger le compte rendu de ses travaux. Cette académie s'affilie à l'Alliance française en 1888 et devient, en 1938, une simple Alliance française qui ne publie que ses comptes rendus annuels.

Après le coup fatal, porté en 1914 par la suppression totale (sous prétexte d'économie) du bilinguisme dans la législation et dans les écoles primaires, que peuvent les Louisianais pour sauver la langue française dans leur pays ? Depuis lors, le français n'est enseigné comme langue seconde qu'à partir de la huitième année. Dans les High schools et à l'université, l'étudiant est libre de choisir, comme langue seconde, l'espagnol ou le français. Plusieurs high schools ont leur club français, sous la direction de professeurs de langue française. Le gouvernement de l'État a fait bâtir dans sa propre université la «Maison française », foyer de culture à la disposition de ceux qui voudront conserver la langue et l'esprit français. Il y a donc lieu d'espérer qu'une élite parlera, lira et écrira toujours le français en Louisiane.

De plus, le français est conservé dans les vieilles familles, particulièrement les familles acadiennes qui forment la majorité de la population française. Ce sont elles surtout qui ont gardé leurs traditions, leur langue et leur foi. Les adultes et les vieil- 
lards parlent en général le français, le dialecte acadien, un langage qui est celui de leurs pères, un français émaillé d'expressions étrangères qui se trouve surtout à Saint-Martinville et aux environs. Les enfants, qui reçoivent leur instruction primaire uniquement en anglais, ne s'abreuvant que de littérature anglaise, parlent parfois français dans la famille, mais cela ne se rencontre pas dans toutes les familles. C'est presque un miracle que toute une génération de vieillards et une autre d'adultes parlent le français sans l'avoir appris à l'école.

Les noirs parlent le patois qu'on appelle le créole; c'est un français tout à fait à eux, qu'ils conserveront peut-être plus longtemps que les autres citoyens de langue française; comme ils ne fréquentent pas les écoles, l'anglais ne s'infiltre que beaucoup plus lentement chez eux.

A l'occasion du deuxième congrès de la langue française au Canada, tenu à Québec, en juin 1937, on a organisé des concours littéraires pour les professeurs et les élèves dans les universités et les high schools de la Louisiane, mettant en vedette le mot d'ordre québécois: «Conservons notre héritage français». Un groupe de langue française fut organisé en Louisiane pour prendre part à ce congrès.

De telles initiatives, comme les voyages de liaison (comme celui qui fut organisé en Acadie en 1939, et celui du Conseil de la Vie française en Amérique qui partit de Québec et Montréal, en janvier 1955) sont de nature à maintenir et à raviver le flambeau. Lors des réceptions officielles organisées à l'occasion du bicentenaire acadien, les étudiants des high schools ont exécuté plusieurs chants en français, avec une parfaite diction française; entre autres: le Réveil de la Louisiane, Ma Normandie, Au clair de la lune, Bonsoir, belle Rosine, BanBambou, Bonjour, mes amis, etc. La plupart des discours officiels ont été prononcés en français. C'est dire que ces fêtes ont tout de même donné lieu à un certain réveil français; espérons qu'il ne se perdra pas, avec le cours des ans, dans une léthargie regrettable. Il est à souhaiter que plus de revues et de journaux français du Québec pénètrent dans le territoire louisianais pour soutenir l'effort accompli et 
empêcher que ne se perde le goût de la culture et du parler français.

Aujourd'hui, la Louisiane fourmille de journaux publiés en langue anglaise, soit 105 hebdomadaires et 21 quotidiens. Ajoutez à cela 47 postes émetteurs de radio qui diffusent en langue anglaise, 41 bibliothèques ( 4 publiques et 37 paroissiales) remplies d'ouvrages en langue anglaise. Le Teche News de SaintMartinville, dans son numéro du 21 janvier 1955, annonçait qu'une bibliothèque paroissiale, avec abonnement gratuit, venait d'ouvrir ses portes pour la population de cette paroisse et celle de Pont-Breaux. On y faisait mention qu'à l'occasion du bicentenaire acadien, les ouvrages traitant de l'histoire locale avaient une grande popularité, et l'on ajoutait que les ouvrages en langue française y étaient encore en nombre limité. Que peut-on espérer pour la culture française dans de telles conditions ?

Il va de soi que la nécessité impérieuse où se trouve le Louisianais, qu'il soit d'origine française, canadienne ou acadienne, de gagner sa vie dans un état saturé d'américanisme ne lui permet guère de sacrifier ses chances d'avancement ou de progrès dans l'unique but de conserver jalousement, par respect des traditions, le legs culturel de ses ancêtres. Trouvera-t-on le moyen de concilier ce qui n'est peut-être pas tout à fait inconciliable?

JEANNE GRÉGOIRE 